\title{
La arquitectura de los pueblos milenarios del altiplano: una configuración femenina y masculina, Jaqi Andino
}

\section{The architecture of the millenary peoples of the altiplano: a female and male configuration, Jaqui Andean}

Pacha Yapucha Yampara Blanco*

\section{Resumen}

Los pueblos ancestrales situados en el altiplano boliviano evidencian una tradición rica en arquitectura con tierra. El sistema de vivienda, parte del patrimonio arquitectónico altiplánico, por la hegemonía de la lógica mercantil capitalista del "desarrollo" y progresismo, se encuentra ignorado y olvidado. Hoy vemos este sistema de viviendas desmoronándose año tras año, sin importar a nadie el contenido cognitivo, histórico y cultural que representan. En él se evidencia la existencia paritaria del muruqu-taru uta (casa de base circular y cuadrangular), como componente principal y particular del sistema de vivienda del Ayllu y la Marka estudiada del altiplano. Al realizar el trabajo de campo y levantamiento de las unidades analizadas, se halló la configuración de tres espacios en el sistema de vivienda: el uta manqha (casa adentro), el uta taypi (centro de la casa) y el uta anqa (entorno y contorno de la casa), donde se plasma la lógica material-espiritual en comple- mento, equidad y cosmo-convivencia armónica, además de presentar una alta sabiduría ecológica ambiental, al utilizar materiales naturales del lugar, pensando en el bienestar de los otros mundos del cosmos.

Palabras clave: Muruqu-Taru uta, patrimonio, construcción con tierra, sistema de vivienda.

\section{Abstract}

The ancestral villages located on the Bolivian highland make an evidence of the wealth of earthen architectural culture existing in the region. The housing system, part of the local architectural heritage, has pitifully been ignored and forgotten, because of the hegemony of the capitalist commercial logic, contributed by the development and progressivism. Today we see this housing system collapsing as time passes by, regardless of its cognitive, historical and cultural content. Within this system, there is evidence

Fecha de recepción: 17 de mayo de 2019 - Fecha de aceptación: 25 de septiembre de 2019

* Arquitecta y Magister Scientiarum en Educación Superior, Universidad Mayor de San Andrés; Facultad de Arquitectura, Artes, Diseño y Urbanismo, Bolivia. Correo electrónico: pyampara.bl@gmail.com 
of the parity of the muruqu-taru uta (house of circular and quadrangular shape), as the main and specific components of the housing system of the Ayllu and Marka studied on the Highlands. During fieldwork, three spaces were found in the housing system: a) the manqha uta (private home space); b) the taypi uta (central home space); c) uta anqa (environment and contour of the home), where the material-spiritual logic of the Aymara culture is embodied in counterpart, equity and harmonious cosmo-coexistence, presenting a high ecological and environmental knowledge, using natural local materials, thinking about the welfare of the other worlds of cosmos.

Keywords: Muruqu-Taru uta, heritage, earthen construction, house system.

\section{Introducción}

Los pueblos tradicionales de los Andes bolivianos presentan una diversidad de técnicas constructivas en sus estructuras con tierra. Muchas de éstas, muestran particularidades en su arquitectura que todavía no han sido estudiadas y que forman parte de un patrimonio arquitectónico ancestral totalmente encubierto, ignorado y olvidado.

Al hacer una mirada retrospectiva y reflexiva, observamos la transformación del contexto y del ambiente en el área rural. La configuración de las construcciones estaba constituida por la paridad de las casas: redondas y cuadradas con sus patios, hechas de materiales naturales como la tierra, esencia del contexto constructivo ancestral del altiplano; y, por ello, las edificaciones con ladrillo y hormigón se desconocían o eran escasas. Hoy en día éstas se propagan cada vez más, dejando a un lado la construcción con materiales naturales y amigables con el medio ambiente.

Es importante notar que las viviendas rurales actuales son una adaptación de lógicas constructivas extendidas desde los centros urbanos, por el contagio de la modernidad, bajo criterios comerciales y materiales de construcción industriales, sin considerar el diseño, la estructura de la vivienda ancestral, el uso de materiales naturales y el horizonte cultural cognitivo de la vivencia de los pueblos milenarios. En todo caso, existen viviendas sociales, con infraestructura educativa, deportiva, etc., principalmente motivadas por las políticas estatales y municipales, las cuales están en función de la oferta de materiales de construcción mercantilistas, con un fuerte impacto ambiental por el proceso de producción y los escombros producidos durante el proceso de construcción o demolición que desplazan los insumos naturales del lugar como la paja, la arcilla y las diversas mezclas de materiales constructivos. Esta extensión y fomento de las políticas estatales y municipales difieren de la visión y lógicas constructivas de los pueblos milenarios donde no solo los materiales son naturales, sino que también existe una cosmo-convivencia con la naturaleza y los otros mundos de vida; una visión y lógica de alta y profunda ecología.

Hoy día, muchas de las viviendas hechas con materiales naturales en el área rural del altiplano boliviano se encuentran abandonadas, deshabitadas y en proceso de desaparición. Carecen de cuidado y conservación. Esto, además de afectar la salud ambiental, altera la simbología cultural de la paridad del ser jaqi (pareja humana/unidad de lo femenino y masculino del mundo andino) en la configuración estructural de la vivienda nativa. La constitución del muruqu-taru uta (casa redonda y cuadrada), forma una unidad donde el muruqu uta (casa de base circular) y el taru uta (casa de base cuadrada/rectangular) representan simbólica y culturalmente la feminidad y la masculinidad, y viceversa, de la paridad del jaqi.

Así como se constituye la nueva pareja, también se cultiva y construye la arquitectura del sistema de vivienda de los ayllus ${ }^{1}$, el cual se encuentra

1 Ayllu: sistema de organización sociopolítico, productivo, territorial y ritual andino que engloba a las jathas (semilla) y comprende las familias del ayllu. La agrupación de ayllus conforma la marka. 
en proceso de desarticulación del sistema social. Este sistema de vivienda ancestral se encuentra en una crisis generada por el choque de dos sistemas y paradigmas, uno de vida: suma qamaña (vivir y convivir bien) y otro anti vida (desarrollo mercantil capitalista).

Esta situación no ha sido abordada por la academia, en tanto olvida la importancia del tratamiento y utilización de los materiales naturales en la construcción de las viviendas ancestrales que brindan bienestar, no solo al ser humano sino también al medio ambiente, por ser orgánicas y fácilmente degradables y tener características de cosmoconvivencia que contribuyen a la salud integral, holística y al pluriverso de mundos bióticos.

En el presente trabajo se pretende analizar la vivienda ancestral del altiplano boliviano, conocer sus lógicas, las técnicas utilizadas y el significado de la representación cultural del sistema de viviendas en redes interactivas del Ayllu Jach'a Chambi, Quriwara Pacajaqi Marka (hoy provincia Gualberto Villarroel, sur del departamento de La Paz, Bolivia).

\section{Metodología}

El sistema constructivo tradicional, en forma y espacio, está relacionado con los cambios experimentados entre los recuerdos de infancia (la vivencia armónica y dinámica del espacio) y lo observado en la actualidad (estructuras y espacios en desuso, abandono), inquietud generada por estudios formales en arquitectura y el interés por las construcciones con tierra. El espacio de trabajo y la población del estudio fueron las redes de sistemas de viviendas del Ayllu Jach'a Chambi.

La metodología utilizada en un inicio fue la entrevista a personas locales para indagar sobre los nombres y significados de los diversos espacios y elementos arquitectónicos. Una vez aclarado este tema se limitó el estudio a 15 áreas domésticas, donde se podría contar con los cuatro elementos característicos que conservaban en su configuración espacial: el muruqu-taru uta (casas de base redonda y cuadrada), más el patio y otros complementos como pirwas (silos) y la qincha (cocina al aire libre) con el q’iri (fogón de arcilla), en su presentación estructural como uta manqha: casa adentro, uta taypi: centro de la casa y uta anqa: contorno y entorno de la casa.

En otra etapa del estudio se realizó un registro fotográfico y gráfico, con levantamiento arquitectónico de los espacios construidos y abiertos en planos arquitectónicos de planta, además de la articulación entre éstos. Con estos datos se realizó un análisis para encontrar patrones de asentamiento que podrían afirmar una lógica y cosmovisión propia del lugar. El trabajo se concluyó con dibujos en perspectiva mediante reconstrucción digital del estado actual de las unidades domésticas analizadas.

Cabe mencionar que el trabajo se realizó en lengua aymara, recuperando las palabras y los significados originales. Posteriormente, se tradujo al español con el ánimo de abarcar dichos significados. Estas palabras aymaras están vigentes y todavía se utilizan.

\section{Antecedentes históricos culturales}

Mediante investigaciones arqueológicas y arquitectónicas en Bolivia y Perú, se ha podido observar la existencia de estructuras circulares y cuadrangulares. Escalante (2018) menciona que "en estos dos sistemas de construcción [...] está más bien, la señal de influencia de las culturas Chiripa y Pukara con su aporte en construcciones de planta cuadrada y las de Wankarani y otras con las edificaciones de planta circular" (p. 276). Lo que nos hace entender que nuestras culturas han trabajado sistemáticamente en este tipo de viviendas.

En el estudio de teledetección realizado en Tiwanaku por Gallego y Pérez (2018) se afirma que "se reflejan en las imágenes obtenidas, las huellas que dejaron grupos de cabañas circulares [...] 
pertenecientes al periodo formativo tardío (siglos I-VI d.C), estos restos visibles son las bases de gran número de construcciones circulares, con medidas muy similares" (p. 89). Más adelante explican:

Se ha observado en Tiwanaku el cambio de diseño constructivo de estas cabañas hacia formas rectangulares, este cambio resulta común en el periodo IV (500-750 d.C. [...]. Este cambio, de estructuras circulares a estructuras cuadrangulares [...] implica el conocimiento referente a las fórmulas de edificación complejas, sobre todo en cuanto al soporte de las cubiertas (p. 93).

Por otro lado, Marussi (1999) afirma sobre los putucos $^{2}$ de Puno que sus "formas esenciales están caracterizadas por sus plantas cuadradas o rectangulares" (p. 93).

En lo que respecta a la valoración espiritualmaterial de las estructuras en las culturas andinas, Javier Lajo (2005) escribe sobre los estudios realizados en la isla de Amantani, Perú. A la plazoleta circular Pachamama le asigna un carácter femenino, iemntras que la cuadrada Pachatata tendría significación masculina, espacios que conforman observatorios astronómicos:

La presencia de esta tipología Par [...] cuadrado y el circular, el 'paterno' y el 'materno' [...] lleva a descubrir el significado simbólico particular de cada uno, nos conduce a entender su simbolismo relacional o vincularidad, vínculos de complementación y proporcionalidad entre las dos figuras geométricas como formulas simbólicas para entender la complejidad de su conjunto, puntos en paridad con el complejo sistema simbólico de la cruz cuadrada del Tiwanaku (pp. 74-75).

En ese sentido se entiende que Pachamama hace referencia al tiempo y espacio de la doble energía material-espiritual de la feminidad y Pachatata

2 Estrutura característica de la cultura Uru, pueblo originario de los territorios circunlacustres del Lago Titicaca, Desaguadero y Lago Poopó de Bolivia y Perú. hace referencia al tiempo y espacio de la doble energía material-espiritual de la masculinidad.

Por otro lado, se debe mencionar que desde hace aproximadamente 50 años muchos jóvenes del lugar han decidido buscar "una vida mejor", trasladándose a los centros urbanos nacionales y extranjeros; éstos al volver a su tierra, en su intención de "mejorar" y "contribuir al desarrollo" han fortalecido la copia arquitectónica y la forma de vida de los centros urbanos, obligando a sus padres y madres -en muchos casos-a ser parte de esta modernidad. Al respecto, la Sociedad Científica Estudiantil de Arquitectura de la Universidad Mayor de San Andrés (FAADU, 2013) menciona que "el abandono de los hábitos tradicionales en el medio rural precedido por varias causas ha tenido una incidencia directa en la progresiva desaparición de la arquitectura vernácula" (p. 11).

Las personas que retornan a las comunidades generan una modificación en la utilización de los materiales de construcción y los espacios. Se cambia el q’iri (cocina de arcilla) por la cocina a gas, la qincha (espacio de elaboración de alimentos) por el espacio de la cocina cerrado, el muruqu-taru uta por cuartos tipo departamento en altura sin importar su orientación; se cambia la qamaña uta (casa) cosmo-convivial por el uta qhatu (casa negocio) ${ }^{3}$ mercantilizado. En muchos casos, estas transformaciones se extienden a la dinámica agrícola y ganadera, es así cómo el arado con yunta (par de toros) se cambia por el uso del tractor, el qalakayu (burro de carga) por las motos y autos que se consiguen de segunda mano. Incluso la forma de alimentación y oferta en los qhatus (ferias) del pueblo han sido alteradas, donde el poder del mercado ha tomado posesión y se pueden conseguir los mismos productos "alimenticios" que se encuentran en los centros urbanos.

3 Ver Qullqan qamaña uta: casa, nido de vivencia con los bienes naturales, la casa-negocio alteña. 


\section{Entrevistas: rescate oral sobre el sistema de viviendas}

Las entrevistas se realizaron a seis personas de la comunidad de las cuales cuatro eran propietarias y las otras ajenas a las viviendas relevadas. Estas entrevistas permitieron comprender a profundidad los pormenores del sistema de vivienda del altiplano.

\section{Contextualización de la Uta: casa, nido, vivienda Aymara}

Cuando hablamos de Uta (casa, en aymara) nos referimos a la casa con espíritu propio donde existe la paridad de las energías materiales-espirituales: japhallani-uta (vivienda material con su propio espíritu ${ }^{4}$ ). La vivienda físico-espacial de las familias aymaras se denomina cosmológica y ritualmente Tapa Kuntur Mamani (Nido de Cóndores y Mamanis). Así también se evoca a los bienes de la vida: quri tapa (nido de oro: valor masculino), qullqi tapa (nido de plata: valor femenino), qullqa tapa (nido de bienes naturales y riqueza/bienes materiales), qamaña tapa (nido de la vivencia y convivencia/bienes inmateriales). todos estos aspectos se extienden desde la gente hacía el pluriverso de mundos bióticos. Resumiendo, en aymara se dice qamasan jaqinakan, qullqanakanpi qamirinakan utapa (casa de la vivencia de la gente con bienes de la vida y riqueza, cultivador de la salud ecológica, ambiental y con energía paritaria propia).

La uta constituye el nido de la red de familias del sistema de vida de los ayllus y markas. Esta red se refleja con la variedad de la topografía territorial (Amazonas 200-500 msnm, valles interandinos $1.000-2.000 \mathrm{msnm}$ y altiplano $3.000-4.000 \mathrm{msnm}$ ) existiendo todo un sistema de nidos interecoló-

4 Es importante mencionar que la vida aymara es altamente ritual en todo sentido.

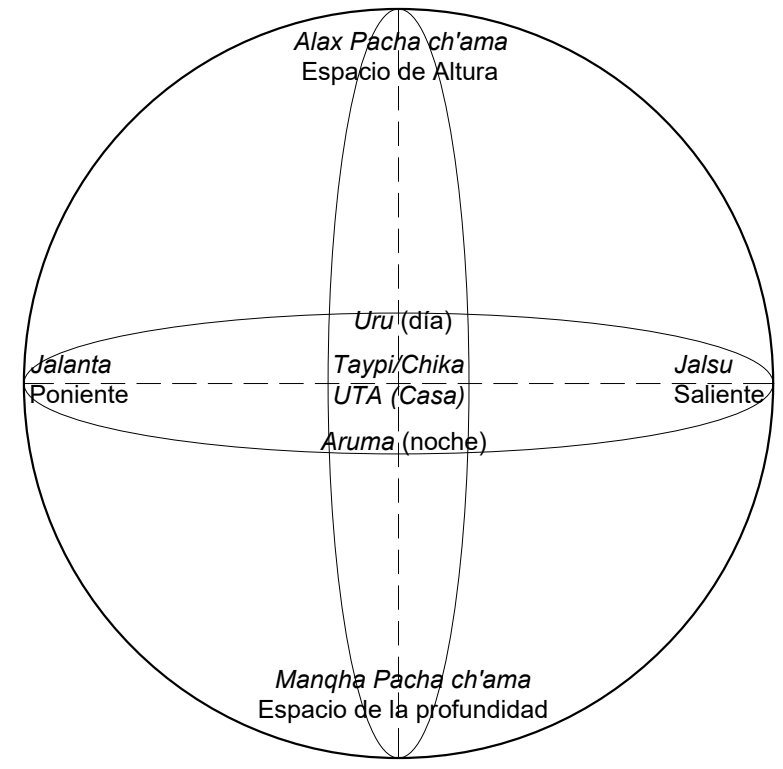

Figura 1. Dinámica energética en el cosmos biótico andino. Fuente: elaboración propia.

gicos en convivencia con el pluriverso de mundos bióticos. Esta relación e interacción se hace en ayni (reciprocidad) con el pluriverso de mundos: la tierra, las wak'as (deidades o lugares energéticos), los animales, las plantas y la gente que hace otro nivel de convivencia de mundos bióticos: taypi uta (casa intermedia). En otro nivel macro está la casa mayor del planeta tierra, constituida por aguas del mar, las cordilleras y la tierra que configuran sistemas de vida acuática y terrestre como partes del ser biótico orgánico natural planetario. En el sentido aymara, $u t a$ (casa) puede tener expresiones, dimensiones y comprensiones distintas y variadas.

A continuación se detallan los elementos de la configuración espacial, su valoración convencional y su desarrollo, según información recogida en las entrevistas. 
Tabla 1. Configuración espacial y función del sistema de vivienda ancestral.

\begin{tabular}{|c|c|c|}
\hline $\begin{array}{l}\text { Espacio } \\
\text { (aymara) }\end{array}$ & Equivalencia & Configuración funcional \\
\hline \multicolumn{3}{|c|}{ Uta Manqha: casa adentro } \\
\hline $\begin{array}{l}\text { Muruqu } \\
\text { uta }\end{array}$ & $\begin{array}{l}\text { Casa de base de } \\
\text { forma circular usada } \\
\text { cotidianamente. Su } \\
\text { mayor uso se da en } \\
\text { juyphipacha (épocas } \\
\text { de heladas) y épocas } \\
\text { de lluvia. }\end{array}$ & $\begin{array}{l}\text { De día es sala de encuentro, comedor de los miembros de la familia y eventual- } \\
\text { mente recibimiento de visitas. De noche es dormitorio donde los miembros de } \\
\text { la familia descansan por género y etariamente ordenados de menor a mayor, } \\
\text { estando los padres en el centro, con tendido y tapado de dos colchas llamados } \\
\text { qamiri/kurji. Metafóricamente representa al vientre materno, es el espacio } \\
\text { que acoge a los habitantes de la vivienda. Se relaciona a la reproducción y } \\
\text { vida. Cuando la mujer daba a luz, descansaba en el muruqu uta, cumpliendo } \\
\text { funciones de hospital. Según las abuelas, la anatomía de la mujer debía ser } \\
\text { resguardada descansando después del parto. Con toda sabiduría exigían que } \\
\text { las madres descansaran y vivieran dentro del muruqu uta durante mínimo } 3 \\
\text { meses y sin ver los rayos solares por la fragilidad en los ojos; sin hacerse soplar } \\
\text { por el viento frío y sin tocar agua, por la fragilidad de la piel y los oídos. } \\
\text { La puerta tiene una orientación hacia la saliente del sol (Este). Contiene un } \\
\text { q'iri (cocina de fogón de arcilla) para épocas de lluvia y frío que se convierte } \\
\text { en estufa para la vivienda y el punto de reunión donde se comparte en familia. } \\
\text { Un elemento importante es el t'uju (orificio pequeño) ubicado al Noreste por } \\
\text { donde penetran los rayos solares. También cumple la función de ventilación y } \\
\text { salida del humo como una chimenea. } \\
\text { En la parte de giro de la puerta, denominado qhiru, se enterraba la placenta de } \\
\text { la wawa (neonato), para que los hijos no se marcharan lejos de la casa y fueran } \\
\text { ordenados. } \\
\text { En algunos casos, se apreciaban también astas de toro arriba de la puerta para } \\
\text { proteger la integridad de los hijos respecto a la salud, defensa y espanto de } \\
\text { malas energías. } \\
\text { Al interior, al fondo y frente a la puerta, había una estrella de forma de chakana } \\
\text { (cruz andina) hecha sobre dos ejes cruzados con fibras de lana de colores de } \\
\text { arco iris. Lo que simbólicamente representa a la Mujer y la Pachamama. }\end{array}$ \\
\hline Taru uta & $\begin{array}{l}\text { Casa de base } \\
\text { cuadrangular; se } \\
\text { usa de despensa y } \\
\text { hospedaje ocasional. }\end{array}$ & $\begin{array}{l}\text { La casa cuadrada generalmente tiene puerta al Este (saliente del sol). Se utiliza } \\
\text { también para habitar, pero la funcionalidad preponderante es despensa y depó- } \\
\text { sito de herramientas agrícolas, materiales de trabajo, alimentos y semillas. En } \\
\text { muchos casos posee uno o dos t'uju, por donde entra la luz. Se utilizaba para } \\
\text { cobijar a las autoridades que se encontraban de visita en los ayllus. En la parte } \\
\text { frontal superior de la puerta se apreciaba una chakana para protección de la } \\
\text { familia igual que en la casa redonda. Simbólicamente representa al varón y } \\
\text { Pachatata. }\end{array}$ \\
\hline \multicolumn{3}{|c|}{ Uta Taypi: el centro de la casa } \\
\hline $\begin{array}{l}\text { Taypi Uta } \\
\text { (anqatuqu) }\end{array}$ & $\begin{array}{l}\text { Patio, sala } \\
\text { abierta: mundo } \\
\text { de interacción } \\
\text { y relación de } \\
\text { convivencia con las } \\
\text { energías naturales: }\end{array}$ & $\begin{array}{l}\text { El patio es un espacio central que articula los otros espacios dentro de } \\
\text { la vivienda ancestral, ahí estaba presente la tapilla (asiento de barro y } \\
\text { protección del muro) conexo al taru uta; también se apreciaba una mesa, } \\
\text { utilizada para las visitas y el akhulli (compartir las hojas de coca). }\end{array}$ \\
\hline
\end{tabular}




\begin{tabular}{|c|c|c|}
\hline & $\begin{array}{l}\text { sol, luna, aire, } \\
\text { viento, lluvia, } \\
\text { recepción de visitas } \\
\text { y acopio de bienes } \\
\text { del pluriverso de } \\
\text { mundos. }\end{array}$ & $\begin{array}{l}\text { El patio es importante porque es un taypi (centro) que conecta las energías } \\
\text { del alax-manqha pacha (espacio de arriba y abajo). } \\
\text { Tiene una puerta llamada 'sawan punku' orientada generalmente al Este. } \\
\text { En algunos casos podía existir más de un patio. }\end{array}$ \\
\hline $\begin{array}{l}\text { Q'iri- } \\
\text { Qincha }\end{array}$ & $\begin{array}{l}\text { Cocina al aire } \\
\text { libre con cerco de } \\
\text { tendido de leña de } \\
\text { t'ula: espacio de } \\
\text { cocción y comedor } \\
\text { de alimentos. }\end{array}$ & $\begin{array}{l}\text { Generalmente se encontraba fuera del patio. Es un espacio que se delimita } \\
\text { de forma semicircular con t'ulas (arbusto endémico) secas de aproxima- } \\
\text { damente } 0.80 \text { m de espesor. Al interior está el elemento más importante, } \\
\text { el q'iri (fogón de arcilla con bosta de burro o paja suave.), con tres "ojos" } \\
\text { y una apertura por donde se alimenta el fuego con leña. Sobre los "ojos" } \\
\text { se ponen las ollas de barro para cocinar. Por estar al aire libre no se tiene } \\
\text { problemas de olores y evacuación de humo. } \\
\text { En algunos casos cuenta con un espacio conexo con el mismo sistema de } \\
\text { delimitación que sirve de comedor, manq'aña qincha. Se encuentra al aire } \\
\text { libre permitiendo el cuidado del ganado en el tiempo de las comidas y la } \\
\text { convivencia con las energías naturales. } \\
\text { En este espacio puede estar el piqaña-wayk'a kiyaña qala (piedra plana } \\
\text { con batan 'tiwiña qala' o piedra redonda 'muruqu qala', dos tipos de } \\
\text { piedra que usan para moler cereales y ají). }\end{array}$ \\
\hline Pirwa & $\begin{array}{l}\text { Depósito, silos } \\
\text { aéreos de granos, } \\
\text { quinua y papas } \\
\text { deshidratadas: } \\
\text { chuñu (mundo } \\
\text { vegetal). }\end{array}$ & $\begin{array}{l}\text { Son depósitos de medidas reducidas, aproximadamente }(1.0 \times 0.6 \mathrm{~m}) \text {, } \\
\text { con dos aperturas laterales libres, una superior (aprox. } 0.30 \times 0.35 \mathrm{~m}) \text { por } \\
\text { donde se introducían los alimentos a una altura } 1.20 \mathrm{~m} \text {, y otra en la parte } \\
\text { inferior por donde se extraían los alimentos depositados. } \\
\text { Existía más de una unidad en cada vivienda, dependiendo de la produc- } \\
\text { ción, cosecha y procesado de alimentos. }\end{array}$ \\
\hline \begin{tabular}{|l|} 
Piluna \\
(Silo)
\end{tabular} & $\begin{array}{l}\text { Depósito de forraje } \\
\text { (mundo vegetal). }\end{array}$ & $\begin{array}{l}\text { Es un depósito que puede tener solo cubierta en un espacio al aire libre, } \\
\text { utilizada para apilar el forraje para el ganado. }\end{array}$ \\
\hline $\begin{array}{l}\text { Wallpa } \\
\text { uta }\end{array}$ & $\begin{array}{l}\text { Casa de gallina } \\
\text { (mundo animal). }\end{array}$ & $\begin{array}{l}\text { El corral de aves o casa de gallinas se encontraba dentro del taypi uta } \\
\text { (patio) para protegerlas de los animales rapaces. }\end{array}$ \\
\hline \multicolumn{3}{|c|}{ Uta Anqa: al contorno y entorno de la casa } \\
\hline $\begin{array}{l}\text { Tanta } \\
\text { luraña } \\
\text { putu }\end{array}$ & $\begin{array}{l}\text { Horno (espacio } \\
\text { de cocción de } \\
\text { alimentos: pan). }\end{array}$ & $\begin{array}{l}\text { Es un espacio que se encuentra fuera del uta taypi (patio), en muchos } \\
\text { casos cercano a la qincha (cocina). Tenía un uso predominante en amaya } \\
\text { phaxina (noviembre) previo a "Todos Santos", para hacer tanta wawas } \\
\text { (pan tradicional para esta fiesta con forma de humano) y asados para la } \\
\text { celebración con los muertos. }\end{array}$ \\
\hline $\begin{array}{l}\text { Uma puju- } \\
\text { quta }\end{array}$ & $\begin{array}{l}\text { Pozo-lagunilla } \\
\text { (mundo tierra: } \\
\text { agua). }\end{array}$ & $\begin{array}{l}\text { El pozo podía ser compartido o individual, generalmente se encontraba } \\
\text { lejano a la vivienda, en algunos casos también se visualizaba la quta (lagu- } \\
\text { nilla) o jawira (río). }\end{array}$ \\
\hline Anu uta & $\begin{array}{l}\text { Casa del perro } \\
\text { (mundo animal). }\end{array}$ & $\begin{array}{l}\text { Su ubicación podía variar entre el uta anqa y uta taypi, eso dependía de la } \\
\text { familia. Muchas veces está como protector de las gallinas. }\end{array}$ \\
\hline Ivija uуu & $\begin{array}{l}\text { Corral de ovejas } \\
\text { (mundo animal). }\end{array}$ & $\begin{array}{l}\text { Antiguamente los corrales eran de t'ulas paradas, amarradas por cuerdas } \\
\text { de pajas o cuero, que se trasladaban de un lugar a otro para abonar el terri- } \\
\text { torio en las anaqas (espacio territorial estacional de usufructo ganadero). } \\
\text { Hoy son espacios grandes generalmente conexos a la vivienda o muy } \\
\text { próximos, de donde se extrae el abono. }\end{array}$ \\
\hline
\end{tabular}




\begin{tabular}{|l|l|l|}
\hline $\begin{array}{l}\text { Waka } \\
\text { chinuña }\end{array}$ & $\begin{array}{l}\text { Lugar de descanso } \\
\text { de los vacunos } \\
\text { (mundo animal). }\end{array}$ & $\begin{array}{l}\text { Es un espacio exclusivo para albergar al ganado vacuno, anexo a ello } \\
\text { pueden estar establos o casuchas de waka qallu (cría de la vaca) para los } \\
\text { terneros recién nacidos o para ordeñar. Tiene a su vez otro espacio de } \\
\text { waka manq'aña, liwaña (comedor de ganado). }\end{array}$ \\
\hline $\begin{array}{l}\text { Uywiri- } \\
\text { samiri }\end{array}$ & $\begin{array}{l}\text { Espacio sagrado } \\
\text { (mundo de las } \\
\text { wak'as). }\end{array}$ & $\begin{array}{l}\text { Es un espacio sagrado inmaterial de illas, uywiris e ispallas (espíritu repro- } \\
\text { ductivo de los ganados y la producción). Podían ser espacios de tolares } \\
\text { lejanos a la vivienda donde se realizaban cha'llas: celebraciones rituales en } \\
\text { épocas especiales como el anata (carnavales). }\end{array}$ \\
\hline
\end{tabular}

Fuente: elaboración propia.

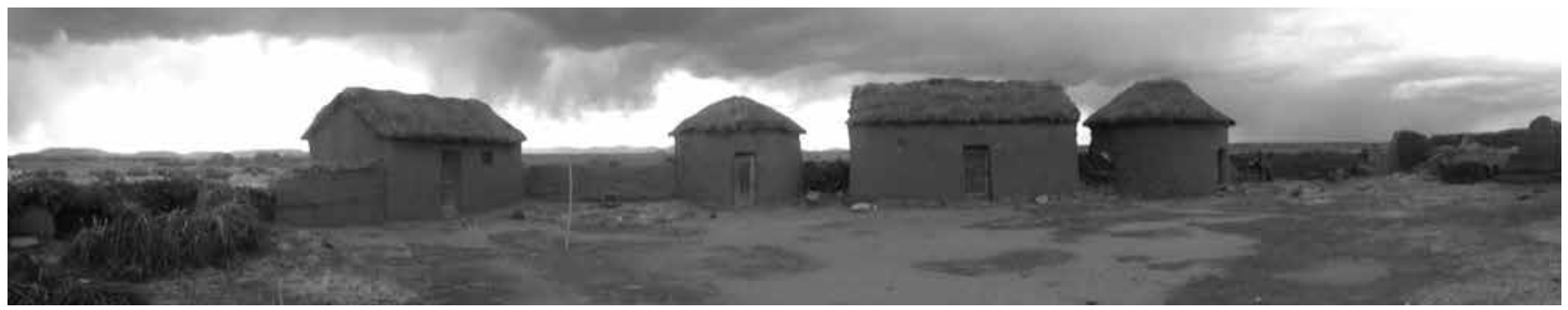

Figura 2. Foto panorámica del complejo sistema de vivienda del altiplano, Levantamiento 1, ubicado en Jalsuri. Fuente: elaboración propia.

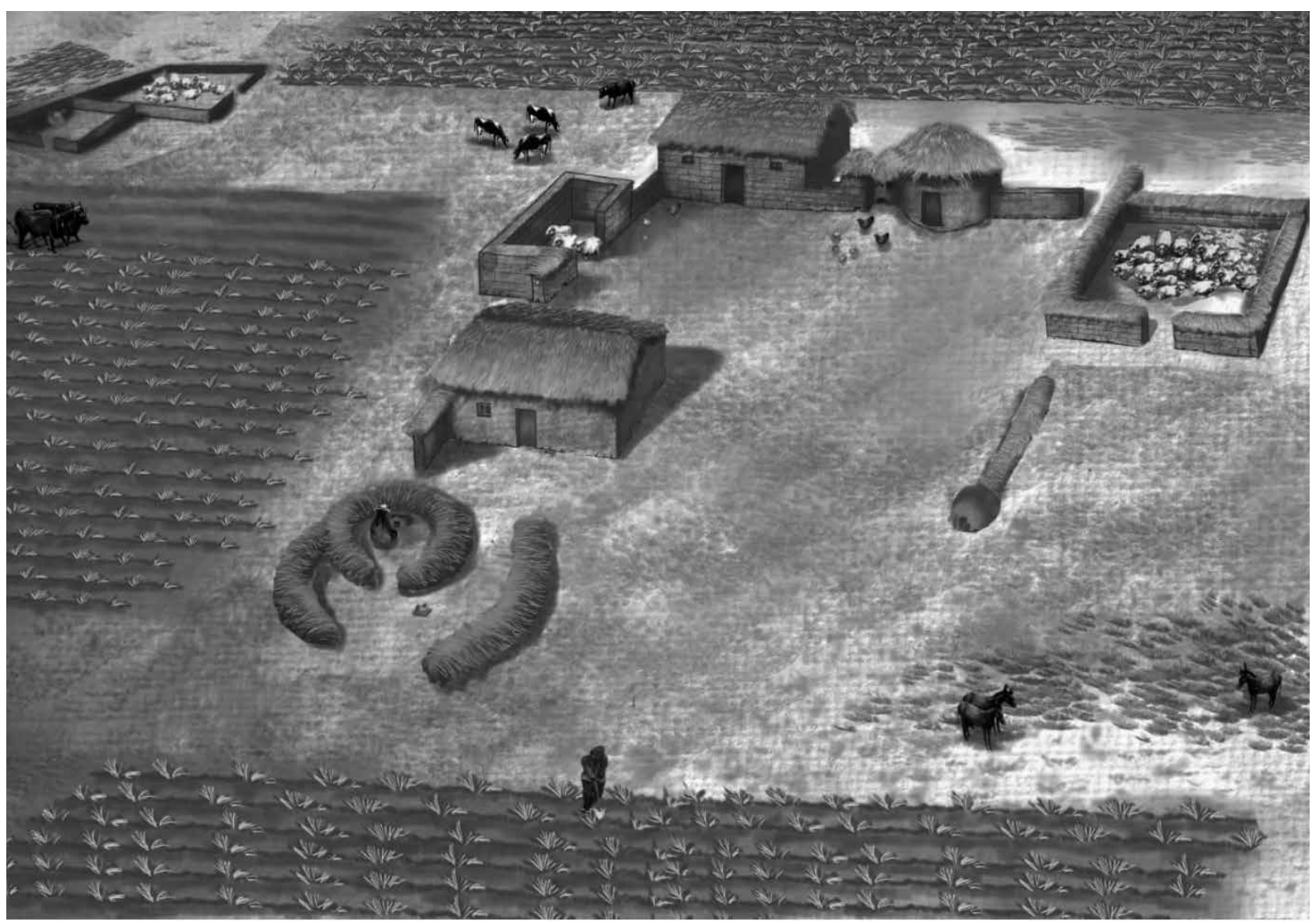

Figura 3. Reconstrucción tridimensional de la disposición del sistema de vivienda, Levantamiento 2. Fuente: elaboración propia. 


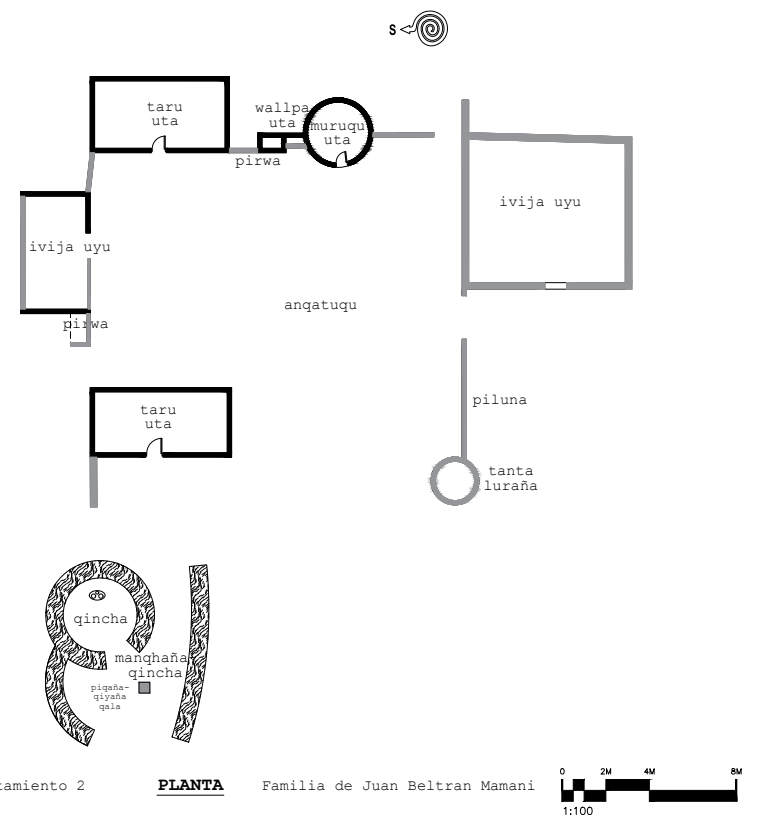

Figura 4. Plano en planta del Levantamiento 2, ubicado en Jalsuri. Fuente: elaboración propia.

\section{Sistema constructivo familiar comunitario}

La manera de construcción del sistema de vivienda ancestral tiene una característica social- ritual en su desarrollo. Existen "maestros de construcción" que entienden y guían en los sistemas y técnicas de construcción, pero lo esencial es la participación de las redes familiares. Ésta se extiende hasta los familiares espirituales como son los padrinos, madrinas y los ahijados y ahijadas, quienes cola- boraban con los aportes de los materiales de construcción naturales de la región y con jornadas laborales.

La nueva pareja preparaba en un 70-80 \% el avance de la construcción; mientras que el restante $20-30 \%$ se complementaba el día programado como parte de la dinámica y práctica del ayni (reciprocidad interactiva), donde se solicitaba la participación de los familiares. Ese día se preparaba una comida comunitaria: apthapi y licores, coca para la ch'alla ${ }^{5}$ y la ofrenda. Los familiares mencionados no llegaban ni participaban con manos vacías, sino que además del aporte con la jornada laboral traían consigo materiales de construcción: palos en cantidades pares, $k^{\prime} i p i$ (atado, cargas) de paja brava para el techado. La premisa era que 'es mejor que sobre a que falte', hecho que se relaciona con el bienestar y el cultivo de los bienes en la familia aymara, como indicador y buen augurio para la tapa (vivienda de la pareja) y familia.

Este sistema de trabajo comunitario en ayni es un enlace permanente y periódico de la red en la vivencia de las familias de las saya-saraqas (comunidad), un compromiso sin documentos escritos ni normas del monismo jurídico, pues aquí vale la palabra y los actos en relación de reciprocidad que se contrae a lo largo del ciclo de vida de las familias.

5 Rociado con alcohol y vino o sangre, se realiza en el piso (boca de la pachamama), muros y otros elementos de la casa.

Tabla 2. Fases rituales de la etapa constructiva del sistema de vivienda.

\begin{tabular}{|l|l|l|}
\hline Fase & Denominativo & Actividades \\
\hline Fase I & Ch'alla a uta qallta & Solicitud de permiso a la pacha para iniciar la construcción de la casa. \\
\hline Fase II & Akhulli & $\begin{array}{l}\text { Descanso compartiendo la hoja sagrada de coca, con lluxta, espacios } \\
\text { donde se decide las actividades futuras en la construcción. }\end{array}$ \\
\hline Fase III & Ch'alla suna tukuya & $\begin{array}{l}\text { Solicitud de permiso a la pacha para aceptación de la nueva casa para que } \\
\text { así tenga durabilidad y consistencia social y material. }\end{array}$ \\
\hline
\end{tabular}

Fuente: elaboración propia. 


\section{Relevamiento del sistema de vivienda}

En la fase de trabajo de campo se realizó el levantamiento arquitectónico de 15 áreas de vivienda en el Ayllu Jach'a Chambi. Los datos obtenidos se expresan en la Tabla 3.

Tabla 3: Relación y muestra de estudio del sistema de viviendas

\begin{tabular}{|c|c|c|c|c|}
\hline Código & $\begin{array}{l}\text { Saya-saraqa } \\
\text { (Comunidad) }\end{array}$ & Descripción de elementos y espacios & $\begin{array}{l}\text { Técnicas } \\
\text { constructivas }\end{array}$ & $\begin{array}{l}\text { Estado de } \\
\text { ocupación }\end{array}$ \\
\hline L- 1 & $\begin{array}{l}\text { Zona Beltrán } \\
\text { (Jalsuri) }\end{array}$ & $\begin{array}{l}\text { - Muruqu-taru uta } \\
\text { - Anqatuqu, qincha con el q'iri, piluna, pirwa, } \\
\text { wallpa uta }\end{array}$ & $\begin{array}{l}\text { Adobe } \\
\text { Tapia } \\
\text { Tierra amasada }\end{array}$ & $\begin{array}{l}\text { Buena y } \\
\text { habitada }\end{array}$ \\
\hline L- 2 & $\begin{array}{l}\text { Zona Beltrán } \\
\text { (Jalsuri) }\end{array}$ & $\begin{array}{l}\text { - Muruqu-taru uta } \\
\text { - Anqatuqu, piluna, pirwa, wallpa uta, iwija uyu } \\
\text { - Qincha con el q’iri, manqaña qincha, tanta } \\
\text { luraña, iwija uyu }\end{array}$ & $\begin{array}{l}\text { Adobe } \\
\text { Tapia } \\
\text { Tierra amasada }\end{array}$ & $\begin{array}{l}\text { Buena y } \\
\text { habitada }\end{array}$ \\
\hline $\mathrm{L}-3$ & Zona Janqu (Jalsuri) & $\begin{array}{l}\text { - Muruqu-taru uta } \\
\text { - Anqatuqu, iwija uyu }\end{array}$ & $\begin{array}{l}\text { Adobe } \\
\text { Tapia } \\
\text { Ladrillo }\end{array}$ & $\begin{array}{l}\text { Buena, no } \\
\text { habitada }\end{array}$ \\
\hline $\mathrm{L}-4$ & $\begin{array}{l}\text { Zona Mamani Jisk'a } \\
\text { Jalsuri (Jalsuri) }\end{array}$ & $\begin{array}{l}\text { - Muruqu-taru uta } \\
\text { - Anqatuqu, piluna, pirwa, wallpa uta } \\
\text { - Qincha con el q'iri, manq'aña qincha, tanta } \\
\text { luraña, iwija uyu, uma puju }\end{array}$ & $\begin{array}{l}\text { Adobe } \\
\text { Tierra amasada }\end{array}$ & $\begin{array}{l}\text { Buena, en } \\
\text { abandono }\end{array}$ \\
\hline $\mathrm{L}-5$ & $\begin{array}{l}\text { Zona Mamani Jisk'a } \\
\text { Jalsuri (Jalsuri) }\end{array}$ & $\begin{array}{l}\text { - Muruqu-taru uta } \\
\text { - Anqatuqu, pirwa } \\
\text { - Jawira }\end{array}$ & $\begin{array}{l}\text { Adobe } \\
\text { Tierra amasada }\end{array}$ & $\begin{array}{l}\text { Mala, en } \\
\text { abandono }\end{array}$ \\
\hline $\mathrm{L}-6$ & $\begin{array}{l}\text { Zona Mamani Jisk'a } \\
\text { Jalsuri (Jalsuri) }\end{array}$ & $\begin{array}{l}\text { - Muruqu-taru uta } \\
\text { - Anqatuqu, piluna, pirwa, wallpa uta, tanta } \\
\text { luraña, quta } \\
\text { - Qincha con el q'iri, manq'aña qincha, waka } \\
\text { chinuña, waka callu }\end{array}$ & $\begin{array}{l}\text { Adobe } \\
\text { Tapia } \\
\text { Tierra amasada }\end{array}$ & $\begin{array}{l}\text { Buena y } \\
\text { habitada }\end{array}$ \\
\hline L- 7 & $\begin{array}{l}\text { Zona Mamani Jisk'a } \\
\text { Jalsuri (Jalsuri) }\end{array}$ & $\begin{array}{l}\text { - Muruqu-taru uta } \\
\text { - Anqatuqu, piluna, pirwa, } \\
\text { - Qincha con el q'iri, iwija uyu. taru uta, uywiri }\end{array}$ & $\begin{array}{l}\text { Adobe } \\
\text { Tapia } \\
\text { Tierra amasada }\end{array}$ & $\begin{array}{l}\text { Mala, en } \\
\text { abandono }\end{array}$ \\
\hline L- 8 & $\begin{array}{l}\text { Zona Mamani Jisk'a } \\
\text { Jalsuri (Jalsuri) }\end{array}$ & $\begin{array}{l}\text { - Muruqu-taru uta } \\
\text { - Anqatuqu, pirwa, piluna }\end{array}$ & $\begin{array}{l}\text { Adobe } \\
\text { Tapia }\end{array}$ & $\begin{array}{l}\text { Buena, no } \\
\text { habitada }\end{array}$ \\
\hline L- 9 & $\begin{array}{l}\text { Zona Mamani Jisk'a } \\
\text { Jalsuri (Jalsuri) }\end{array}$ & $\begin{array}{l}\text { - Taru uta } \\
\text { - Anqatuqu, wallpa uta, pirwa } \\
\text { - Qincha con el q'iri, manq'aña qincha, quta, taru } \\
\text { uta }\end{array}$ & $\begin{array}{l}\text { Adobe } \\
\text { Tapia } \\
\text { Tierra amasada }\end{array}$ & $\begin{array}{l}\text { Buena, no } \\
\text { habitada }\end{array}$ \\
\hline $\mathrm{L}-10$ & $\begin{array}{l}\text { Zona Mamani Jisk'a } \\
\text { Jalsuri (Jalsuri) }\end{array}$ & $\begin{array}{l}\text { - Taru uta } \\
\text { - Anqatuqu, wallpa uta, pirwa } \\
\text { - Qincha con el q'iri, manq'aña qincha, pirwa, } \\
\text { iwija uyu }\end{array}$ & $\begin{array}{l}\text { Adobe } \\
\text { Tapia } \\
\text { Tierra amasada }\end{array}$ & $\begin{array}{l}\text { Buena y } \\
\text { habitada }\end{array}$ \\
\hline
\end{tabular}


La arquitectura de los pueblos milenarios del altiplano: una configuración femenina y masculina, Jaqi Andino

\begin{tabular}{|c|c|c|c|c|}
\hline L- 11 & Jach'a Yampara & $\begin{array}{l}\text { - Muruqu-taru uta } \\
\text { - Anqatuqu, piluna, pirwa, wallpa uta }\end{array}$ & $\begin{array}{l}\text { Adobe } \\
\text { Tapia } \\
\text { Tierra amasada } \\
\end{array}$ & $\begin{array}{l}\text { Regular y } \\
\text { habitada }\end{array}$ \\
\hline L- 12 & Jach'a Yampara & $\begin{array}{l}\text { - Taru uta } \\
\text { - Anqatuqu, pirwa solo el q'iri de la qincha, establo } \\
\text { - Uma puju }\end{array}$ & $\begin{array}{l}\text { Adobe } \\
\text { Tierra amasada } \\
\text { Ladrillo } \\
\end{array}$ & $\begin{array}{l}\text { Regular, no } \\
\text { habitada }\end{array}$ \\
\hline L- 13 & Jach'a Yampara & $\begin{array}{l}\text { - Taru uta } \\
\text { - Anqatuqu, } \\
\text { - Establo }\end{array}$ & $\begin{array}{l}\text { Adobe } \\
\text { Tapia } \\
\text { Ladrillo } \\
\end{array}$ & $\begin{array}{l}\text { Buena, no } \\
\text { habitada }\end{array}$ \\
\hline L- 14 & Jach'a Yampara & $\begin{array}{l}\text { - Muruqu-taru uta } \\
\text { - Anqatuqu } \\
\text { - Qincha con el q'iri, tanta luraña } \\
\end{array}$ & $\begin{array}{l}\text { Adobe } \\
\text { Tapia } \\
\text { Tierra amasada } \\
\end{array}$ & $\begin{array}{l}\text { Regular, no } \\
\text { habitada }\end{array}$ \\
\hline L- 15 & Jach'a Llujlla & $\begin{array}{l}\text { - Muruqu-taru uta } \\
\text { - Anqatuqu, piluna, wallpa uta } \\
\text { - Qincha con el q'iri, manq'aña qincha, iwija uyu }\end{array}$ & $\begin{array}{l}\text { Adobe } \\
\text { Tapia } \\
\text { Tierra amasada } \\
\end{array}$ & $\begin{array}{l}\text { Buena y } \\
\text { habitada }\end{array}$ \\
\hline
\end{tabular}

Fuente: elaboración propia.

Tabla 4. Técnicas constructivas del sistema de vivienda altiplánico

\begin{tabular}{|c|c|c|}
\hline $\begin{array}{c}\text { Elemento } \\
\text { constructivo }\end{array}$ & Técnicas constructivas y variantes & Imagen \\
\hline $\begin{array}{l}\text { Cimiento y } \\
\text { sobrecimiento }\end{array}$ & $\begin{array}{l}\text { Piedra con mortero de Muro sobre el terreno } \\
\text { barro }\end{array}$ & \\
\hline \multirow[t]{4}{*}{ Muro } & Ch'ampas & \\
\hline & Adobes, con mortero de barro & \\
\hline & Tapia & \\
\hline & Ramas de t'ulas secas & \\
\hline $\begin{array}{l}\text { Revoques y termi- } \\
\text { naciones }\end{array}$ & $\begin{array}{l}\text { Revoque de barro con Grueso y fino } \\
\text { tierra del lugar y tierra } \\
\text { blanca }\end{array}$ & \\
\hline Techos, cubiertas & $\begin{array}{l}\text { Cubierta de paja emba- Solo paja } \\
\text { rrada (tajta) }\end{array}$ & \\
\hline Pisos & $\begin{array}{l}\text { Piso de tierra nivelado } \\
\text { y apisonado }\end{array}$ & \\
\hline
\end{tabular}

Fuente: elaboración propia. 


\section{Las técnicas constructivas registradas en el sistema de vivienda del altiplano}

Los pueblos ancestrales milenarios de los Andes han utilizado una variedad de técnicas constructivas con tierra, dando solución constructiva según el territorio donde habitaban, tal como se evidencia en la Tabla 4.

En su complejo sistema de vivienda se ha evidenciado la utilización de más de una técnica constructiva de tierra, que forma parte de su conocimiento ancestral. A continuación se mencionan algunas de ellas.

\section{a. La tierra apilada con posterior perfilado $^{6}$}

Esta técnica constructiva ha sido utilizada para el q'iri, fogón de arcilla, que ha sido modelado para conformar la pieza final. Inicialmente se recoge la tierra arcillosa, se prepara la mezcla de tierra que muchas veces contiene bosta de burro o vaca. Se amasa hasta llegar a un estado plástico, con el que se va formando la base del q'iri, aproximadamente de unos $60-70 \mathrm{~cm}$ de largo y unos $40-50 \mathrm{~cm}$ de ancho. Al crecer en altura, en la parte delantera se conforma una apertura de unos $20 \mathrm{~cm}$ de diámetro por donde se alimentará el fuego.

Para lograr la forma se utiliza una estructura interna de madera o restos cerámicos. La altura final es de $40 \mathrm{~cm}$. En la parte superior se conforman los dos "ojos" de unos $15 \mathrm{~cm}$ de diámetro, donde se dispondrán las ollas de barro. Algunos diseños están conformados por tres "ojos". Estos se forman gracias a una barra central de madera que separa los dos ojos principales que luego se moldean con la mezcla de tierra. El tercer "ojo" va en la parte posterior para tener mejor manipulación de las llamas del fuego o para reposar alguna olla.

6 Término resultante del proyecto COREMANS (Mileto y Vegas, 2017).

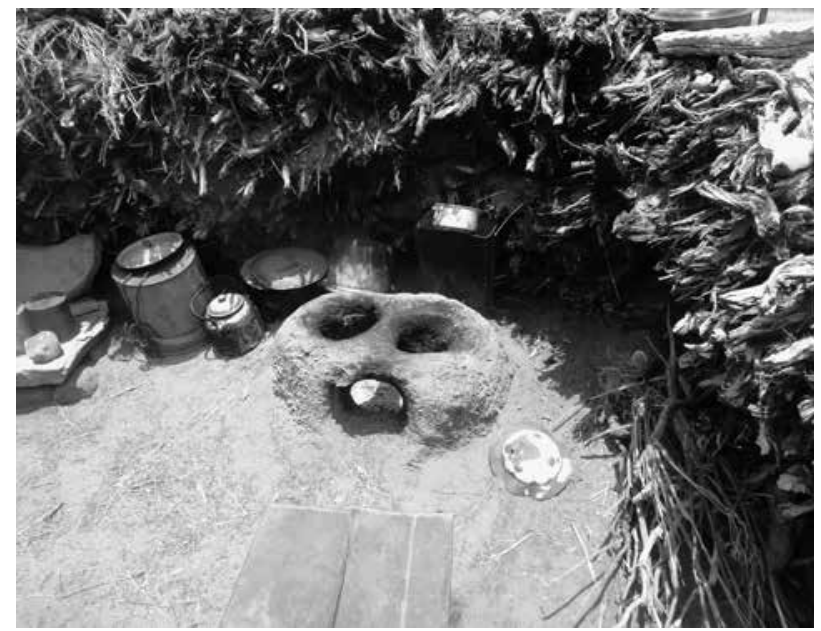

Figura 5. Disposición del q’iri (fogón de arcilla) de dos "ojos" en la qincha, se puede apreciar que la apertura por donde se alimenta el fuego es totalmente circular, forma que se conseguía con restos de cerámica en desuso. Fuente: elaboración propia.

\section{b. Tierra apilada en piezas: piezas con vegetación (ch'ampa)}

Esta técnica ha sido una de las más utilizadas en el altiplano, sobre todo cerca de Oruro. Debido a las condiciones secas del territorio desarrollaron la técnica cortando la tierra que contenía raíces, conocidas como champa, sacadas del suelo con follaje nativo que lo llaman chiji, que al ser combinadas con $n^{\prime} i q i / g r e d a$ arcillosa, eran útiles para la construcción. En lo posible las ch'ampas se hacían con poco salitre y eran cortadas y preparadas después de la época de lluvias.

Esta técnica fue utilizada en los cercos que delimitaban la vivienda. En algunas muruqu utas se han utilizado para conformar el muro. Primero se trazaba el radio con una cuerda donde se iniciaba la construcción. Paralelamente se trazaba un círculo del mismo radio en el lugar donde se extraían las ch'ampas. Éstas debían poseer la misma curvatura para no tener problemas con las aristas al momento de construir y para aplicar el revoque. Las dimensiones eran aproximadamente de $30 \mathrm{~cm}$ de largo externo, $20 \mathrm{~cm}$ de ancho y unos $15 \mathrm{~cm}$ de alto. 


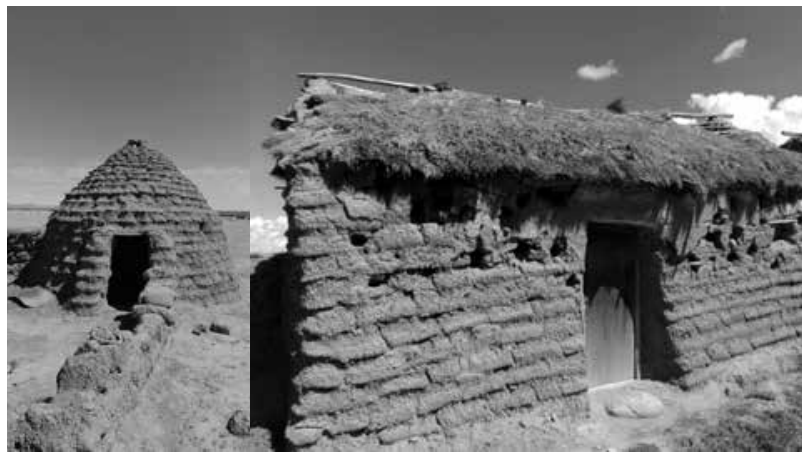

Figura 6. Putuku en Qulqiamaya construido con ch'ampas, donde los muros continúan para conformar el cerramiento del elemento. Muro del taru uta en Jach'a Chambi construido con ch'ampas, por la pérdida del revestimiento se observa la disposición de las piezas con las raíces hacia arriba. Fuente: elaboración propia.

Cerca al ayllu J'acha Chambi se encuentra el pueblo de San José donde se ha evidenciado la utilización de la ch'ampa para la conformación de los putukus, similares a los Chipayas. Éstos no presentan estructura de cubierta, sino se cierra con las mismas ch'ampas. Probablemente, en el lugar no disponían de piezas de madera para conformar el techo.

También se ha utilizado en el taru uta para los muros que fue posible observar en los taru utas cercanos al área de estudio, que se encuentran deteriorados y sin revoques exteriores. En estos casos las piezas de champa tienen las mediadas de $30 \mathrm{~cm}$ de largo, $20 \mathrm{~cm}$ de ancho y $12-15 \mathrm{~cm}$ de alto. En la mayoría de estas unidades al igual que en los muruqu utas no existen cimientos. Se construía sobre el nivelado de la misma tierra.

\section{c. Piezas moldeadas}

\section{Adobes}

Los adobes son piezas moldeadas que requerían un conocimiento específico para su elaboración sin desperfectos; la selección de arcilla con arena (tierra local) y el tipo de paja (sicuya ichu), el amasado y la cantidad de agua exacta. Incluso hay que hacer dormir la mezcla un día lo cual tiene su

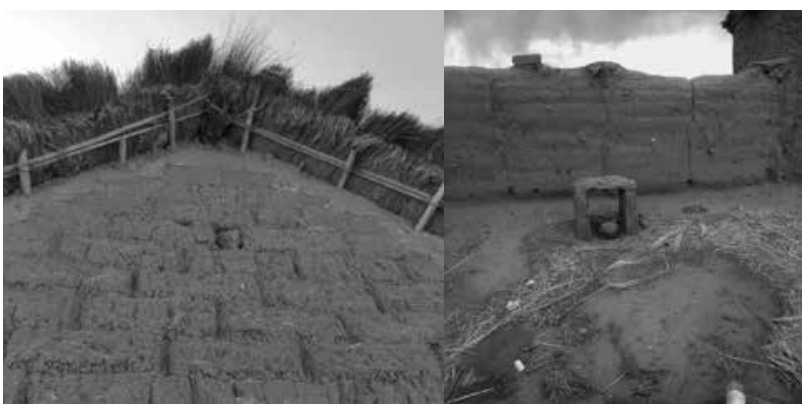

Figura 7. Muro de adobe en el taru uta; tapia en el uta anqa. Fuente: elaboración propia.

explicación lógica, ya que las partículas al estar en contacto con el agua por mayor tiempo se lubrican y la adherencia es de mejor calidad. El secado de la pieza de adobe debe ser de 7 a 15 días volcándolos de lado a lado sin luz directa. Hoy los constructores contemporáneos recomiendan realizar el secado en semi sombra.

La técnica del adobe ha sido utilizada en muchos espacios de la vivienda ancestral del altiplano. Podemos verla en muchos muruqu-taru utas, en el wallpa uta, en algunos iwija uyu, en las pirwas, hasta en algunos muros de cerco.

\section{Tapia}

La técnica de la tapia es muy utilizada para el muro de cerco del espacio uta anqa: centro de la casa. Las piezas miden $100 \mathrm{~cm}$ de largo, $60 \mathrm{~cm}$ de alto y 35-40 de ancho. El proceso inicia excavando tierra del lugar, el cual puede ser mezclado con paja menuda (sicuya ichu) para mejorar el trabajo de la tapia a tracción, ya que la tierra de la zona es muy arenosa. La mezcla en estado húmedo se hace dormir un día mínimamente para homogenizar la mezcla, tener ya preparada gran cantidad de tierra y avanzar en el trabajo de construcción.

El tipo de tierra existente en el ayllu es la arenosa. En algunos espacios existen vetas de tierra más arcillosa del mismo color, lo que mejora su composición y condiciona la consistencia de la tapia. Muchas de las construcciones hechas con tapia están erosionadas en la base por humedad, por capilaridad y poco mantenimiento. 


\section{Morteros y revocos}

En el complejo sistema de viviendas ancestrales los revoques han sido realizados en el interior y el exterior de los muros, de 1,5-2,5 cm de espesor respectivamente. Se preparaba la arcilla con arena, paja suave o bosta de burro desmenuzada, adecuadamente mezclada llegando a un estado viscoso. Se hacía dormir por una, dos y hasta tres noches continuas (lubrificación de arcillas) y de esa manera se tenía una masa consistente y resistente a las heladas, el sol y las lluvias.

El revoque interior es del mismo color de la tierra del lugar, mientras que el revoque exterior en muchas unidades de vivienda es de color blanco. $\mathrm{Si}$ bien, en el lugar no existe tierra blanca, es de suponer que se ha logrado traer de lugares próximos, ya que cerca al Ayllu J'acha Chambi se encuentra la comunidad Jankohota (casa blanca) donde existen vetas de tierra arcillosa blanca. Un ayllu más lejano, Janko Marka (pueblo blanco), es conocido por tener tierra arcillosa blanca.

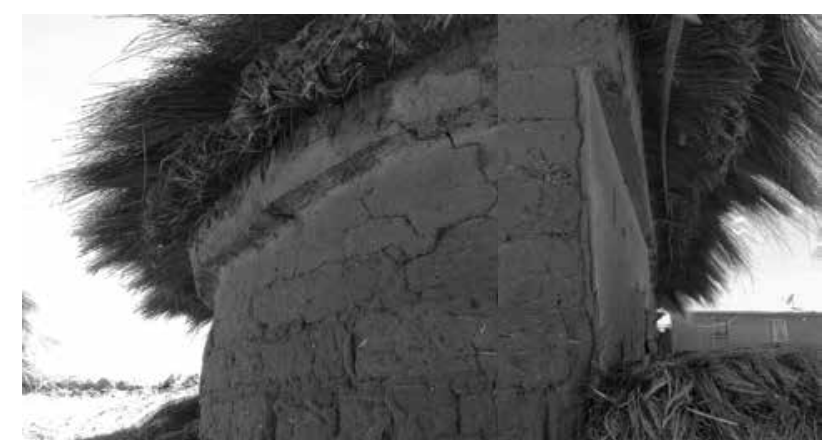

Figura 8. Revoque exterior de color blanco en la parte superior del muruqu uta. Espesor del revoque exterior de color blanco adherido al muro de adobe del taru uta. Fuente: elaboración propia.

\section{La comprensión de la casa desde la cosmología andina}

Los análisis realizados demostraron que el sistema de viviendas del altiplano boliviano presenta características singulares e importantes en su configuración y disposición espacial. Se puede observar una adaptación a las extremas condiciones climáticas generando espacios habitacionales más protegidos para los seres humanos y los animales domésticos. Muestran un profundo conocimiento y sensibilidad hacia la naturaleza, pero también de la vida espiritual dando consistencia complementaria a la materialidad.

Los materiales utilizados en las construcciones son totalmente de origen local, hábilmente trabajados y con una huella de carbono muy baja, sin generar residuos sólidos y contaminantes para una convivencia armónica e implican un impacto mínimo en el frágil ecosistema altiplánico. Más bien la lógica del sistema de vivienda aporta en la fertilidad del suelo, por la rotación de los cultivos y los pastoreos, dejando descansar a la tierra. Tanto la construcción como el mantenimiento era un acto de ritualidad en el que se comprendía que la casa también tiene vida y debe ser cuidada igual que los otros seres queridos. Esto se refleja en el sentido de la construcción del hogar por la nueva pareja, iniciando su cosmo-conviviencia con el pluriverso de mundos bióticos.

En la adaptación climática se puede apreciar el uso de materiales nobles como la tierra y la paja, que son térmicos y regulan las temperaturas extremas del exterior, generando condiciones favorables en el interior; por la misma razón no había ventanas; por los escasos recursos del territorio en el día se ocupaba el patio para los quehaceres. Ahí uno se calentaba en el sol, mientras que los muros protegían contra los constantes vientos. No se ocupaba el interior de las estructuras para las actividades diurnas, aprovechando la luz del día afuera, en el patio. En la intemperie protegida del patio se desarrollaba también la vida social. En el interior existía la vida privada, el descanso. Esta manera de organización permitía una relación y conexión directa y fluida con el pluriverso de mundos, protegiendo los animales y los cultivos, así como con el cosmos.

Las familias participaban de la celebración de emulación ritual de las energías de la vida a la nueva casa, como parte del sistema de celebración. Se evocaba a las wak'as (deidades) del lugar 
y la casa, donde genéricamente se diferencia a las energías espirituales de la vivienda y los bienes? La celebración se realizaba con la ch'alla, que es parte de la tecnología ritual. Tiene su inicio, su desarrollo y su terminación. Al invocar a las deidades se estaría invitando por medio del ayni un plato de comida espiritual (mesa ritual) que se alcanza a las w'akas, uywiris, samiris (deidades de emulación de las energías espirituales y consagración a la materialidad de las cosas). En este ritual circula la coca a través de akhullis comunitarios, una manera de convivir, respetar y recordar a las energías de los antepasados y la Pacha.

Aquí, muruqu-taru (redondo-cuadrado) es la representación simbólica de la unidad compuesta por la mujer y el hombre en equidad del ser jaq $i$ en la vida. Esta unidad vive de manera interactiva y equitativa por medio el ayni. Juntos construyen el nido de la gente (el hogar) que 'saben vivir bien' en el mundo de la gente y 'saben con-vivir bien' con el pluriverso de mundos bióticos.

El 'saber convivir bien' con el pluriverso de mundos implica considerar al mundo de la gente en condición de equidad respecto de los otros, ni más ni menos. Simón Yampara (2016) explica la existencia de cinco mundos "Uraqi: mundo de la tierra; Japhalla, mundo de las wak'as (deidades); Uywa, mundo animal; Yapu, mundo vegetal y Jaqi, mundo de la gente" (p. 145). Entre ellos se practica la relación recíproca por medio de ceremonias rituales. Eso es considerado como Qamaña Uta (convivencia con el pluriverso de mundos) en el cosmos biótico.

Por otro lado, el muruqu, el círculo, es la concentración de la energía en el taypi (centro) dando ciclicidad de la energía de la vida. El taru, el cuadrado, es la concretización de la chakana (las cuatro estrellas de la cruz del sur). Ahí se

7 En aymara la vivienda se denomina Tapa, Kuntur Mamani y los silos de los bienes naturales y riqueza Quri Tapa Qulqi Tapa, Qullqa Tapa y de riqueza, bienes de la vida natural en general evocan a Chuqil Qamir Wirnita (vivencia y convivencia de los bienes de la vida).

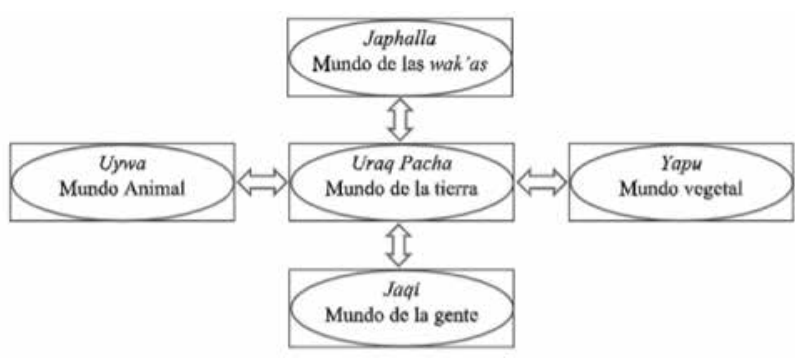

Figura 9. Comunidad biótica para la Qamaña. En el mundo andino se considera que todo tiene vida y todo en la vida es ayni. El Suma qamaña (vivir y convivir bien en armonía) implica el respeto equitativo entre los cinco mundos de vida, entendidos como el pluriverso de mundos bióticos. Fuente: elaboración propia.

concentran y se reproducen los "Cuatro ángulos de la Tierra" 8 que hace referencia a Tiwanaku como herencia cultural ancestral.

\section{Conclusiones}

Al observar el altiplano durante el proceso de estudio de las construcciones y designaciones de espacios en su totalidad, se revela un complejo sistema de vivienda humano en convivencia con otros mundos: el animal, vegetal, las wak'as y la tierra. Esto nos hace entender que la vivienda ancestral va más allá de la visión y acción antrópica, llegando a tener alcance cosmo-convivial, que demanda un diseño arquitectónico diferenciado.

Otra característica general es la paridad del muruqu-taru uta casa redonda-cuadrada, que simbólica culturalmente representa y expresa el género interactivo entre la feminidad (redonda) y la masculinidad (cuadrada). Estas casas conformadas en paridad se orientan al Este para gestionar la energía de la luz y calor del sol. El patio también orientado al este, y conforma una sala a cielo abierto construida para compartir y convivir familiar y comunitariamente.

8 En la cosmovisión andina, los "Cuatroángulos de la Tierra" hacen referencia a los cuatro territorios del Tawantinsuyo, así como a los cuatro puntos cardinales. 
En este trabajo se han recogido tres criterios en el sistema de vivienda: Uta manqha, casa adentro, el espacio privado; Uta taypi, la parte central de la casa, que articula la casa adentro y el entorno, contorno de la casa; y Uta anqa, la parte del entorno y contorno de la casa. La dinámica organizativa de las unidades domésticas a un nivel mayor dentro del ayllu, también está en función de la orientación del movimiento del sol, el urin-saya (espacio de la saliente del sol), el aran-saya (espacio del poniente del sol), articulados interactivamente por la marka (pueblo).

Otro aporte significativo es que la construcción de la casa está relacionada con la constitución de la jaqicha (institucionalización de las nuevas parejas), en la lógica de la autososteniblidad el sistema de la construcción de las viviendas redonda-cuadrada se realiza entre los familiares. Los propietarios hacen preparar una comida especial con la carne de la wilancha (consumo de celebración ritual), que en los hechos se convierte en un ayni familiar próximo y espiritual de los participantes, acción y bienes que son devueltos a su turno cuando las otras familias lo necesitan.

El diseño y la implementación de la arquitectura del sistema de vivienda ancestral de los ayllus y markas andinos tiene una orientación de vida, antes que una mera expresión material y estética. Es una respuesta cultural a sistemas de organización de la vida y sistemas del pluriverso de mundos bióticos. Así también, un trabajo que merece mayores estudios en profundidad, ya que se viene desmoronando por lógicas de desarrollo y modernización del sistema mercantil capitalista, los cuales son una clara expresión de paradigmas anti vida y del espejismo de extensión de modelos urbanos occidentales, altamente contaminantes de la salud ambiental y bienestar del ecosistema territorial de la convivencia del pluriverso de mundos bióticos.

\section{Referencias bibliográficas}

Escalante, J. (2018). La Historia antes de la Historia en Bolivia. La Paz, Bolivia: CIMA, 2da Edición.

FAADU (2011). Registro Documental de Arquitectura Vernácula. Sociedad Científica de Estudiantes. La Paz, Bolivia: CDA.

Gallego, I. y Pérez, M. (2018). Tiwanaku entre el cielo y la tierra. La Paz, Bolivia: UNESCO.

Lajo, J. (2005). Qhapaq Ñan, La ruta Inka de Sabiduría. Lima, Perú: Amaro Runa.

Marussi, F. (1999). Arquitectura Vernacular: Los Putucos de Puno. Lima, Peru: Universidad Ricardo Palma.

Mileto, C. y Vegas López, F. (2017). Coremans proyect. Criterios de intervención en la arquitectura de tierra. Madrid: Ministerio de Educación, Cultura y Deporte de España.

Proyecto Coremans (2017). Criterios de intervención en la arquitectura de tierra. Madrid, España: Ministerio de Educación, Cultura y Deporte.

Yampara, P. (2015). La Arquitectura cosmoconvivial Andina encubierta. 1ra revista cientifica de la carrera de Arquitectura. El Alto, Bolivia: UPEA.

Yampara, S. (2016). Suma Qama Qamaña. Paradigma cosmobiótico Tiwanakuta. La Paz, Bolivia: Qamañ Pacha.

Yampara, P. (2018). MURUQU- TARU UTA: Un sistema de vivienda de Jaqis que saben vivir y convivir bien' con el pluriverso de mundos en el cosmos biótico. Tesis de maestría a defender. UPEA El Alto, Bolivia. 